\title{
Immune interaction map of human SARS-CoV-2 target proteins: implications for therapeutic avenues
}

Karthikeyan Subbarayan

Martin-Luther-University Halle-Wittenberg https://orcid.org/0000-0002-5140-0253

Kamatchi Ulagappan

Martin-Luther-University Halle-Wittenberg https://orcid.org/0000-0002-0396-0731

Claudia Wickenhauser

Martin-Luther-University Halle-Wittenberg https://orcid.org/0000-0002-1807-5575

Barbara Seliger ( $\sim$ barbara.seliger@uk-halle.de)

Martin-Luther-University Halle-Wittenberg https://orcid.org/0000-0002-5544-4958

\section{Research Article}

Keywords: SARS-CoV-2, therapeutics, immune gene, bioinformatics

Posted Date: August 7th, 2020

DOI: https://doi.org/10.21203/rs.3.rs-54541/v1

License: (9) This work is licensed under a Creative Commons Attribution 4.0 International License. Read Full License 


\section{Abstract}

Background There exists increasing evidence that people with preceding medical conditions, such as asthma, diabetes, cancers and heart disease, have a higher risk of infection with SARS-CoV-2 and are more vulnerable to severe disease.

Methods To get insights into the role of the immune system upon COVID-19 infection, 2811 genes of the gene ontology term "immune system process GO: 0002376" were selected for analyses. The immune system genes potentially co-expressed with the human targets of SARS-CoV-2 (HT-SARS-CoV-2) ACE2, TMPRSS2 and FURIN were determined in tissue samples from patients with cancer and diabetes mellitus. The network between HT-SARS-CoV-2 and immune system process genes was analyzed based on functional protein associations using STRING. In addition, STITCH was employed to determine druggable targets.

Results DPP4 was the only immune system process gene, which was coexpressed with the three HTSARS-CoV-2 genes, while eight other immune genes were at least co-expressed with two HT-SARS-CoV-2 genes. STRING analysis between immune and HT-SARS-CoV-2 genes plotted 19 associations of 8 commonly networking genes in mixed healthy (323) and cancer (11003) tissues in addition to normal (87), cancer (90) and diabetic (128) pancreatic tissues. Using this approach, three druggable connections between HT-SARS-CoV-2 and immune system process genes were identified. They include positive associations of ACE2 - DPP4 and TMPRSS2 - SRC as well as a negative association of FURIN with ADAM17. Furthermore, the 16 drugs were extracted from STITCH (score <0.8) with 32 target genes.

Conclusions This bioinformatics pipeline identified for the first time an immunological network associated with COVID-19 infection thereby postulating novel therapeutic opportunities.

\section{Background}

Coronaviruses (CoV) are a large family of viruses that cause illnesses ranging from a common cold to more severe diseases. A novel coronavirus (nCoV named SARS-CoV-2) belonging to this family was identified in 2019 in China and in March 2020, the WHO announced the COVID-19 infection as a worldwide pandemic. As of the 3 Jul 2020, more than 10.8 million cases of COVID-19 infections have been reported in more than 188 countries and territories, causing in more than 521,000 deaths.

SARS-CoV-2 is broadly distributed in humans and other mammals (1) and has a high sequence similarity to SARS-CoV and MERS-CoV (2). Recent studies suggest that SARS- CoV-2 utilizes the angiotensinconverting enzyme II (ACE-2) receptor $(3,4)$, MERS-CoV the dipeptidyl peptidase-4 (DPP4/CD26) as an entry (5). The transmembrane serine protease 2 (TMPRSS2) cleaves and activates the viral spike glycoproteins, which facilitates virus-cell membrane fusions. SARS-CoV-2 requires both the ACE2 receptor and TMPRSS2 expression for protein priming to enter the cell (6). FURIN, a protease present in many human organs, recognizes and activates a specific site on the SARS-CoV-2 spike protein facilitating a tighter binding to the ACE-2 receptor. The increased cleavage activity of this protease was suggested to 
diminish viral recognition by neutralizing antibodies and by activating the SARS spike protein for virus cell fusion (7) thereby facilitating the active binding of SARS-CoV-2 through the ACE2 receptor, which is a risk factor for a more severe COVID-19 infection $(6,8)$.

However, there are still several open questions: Why is SARS-CoV-2 spreading faster than its two ancestors? Why is SARS-CoV-2 more lethal? How does the immune system respond to SARS-CoV-2? Recent publications reported differences in their genome structure (9) and immunological responses next to inflammation (10) (11). The viral entry has been shown to cause a cytokine storm, which can attack lung cells (12). This results in fluid filling of the air sacks and alveolar cell apoptosis causing respiratory insufficiency, ultimately leading to death (13). Currently, some approaches are ongoing to develop potential vaccines and therapeutic agents targeting SARS-CoV-2 or host cell components, e.g., host cell receptors, necessary for viral replication. The complete knowledge of interactions between proteins in a given cell represents essential milestones towards a full description of cellular mechanisms and functions (14). The gene ontology term (GO: 0002376) consists of genes involved in the development or functioning of the immune system and organismal system for calibrated responses to potential internal or invasive threats.

The outbreak of SARS-CoV-2 is an ongoing global public health crisis. The expedition of clinical trials for various potential therapeutic drugs is essential to evaluate their efficacy. Patients' demographic data and their past medical history are crucial for the development of an effective therapeutic plan. The demographic data from Italy showed that among 3000 reported COVID-19 cases, $20 \%$ of the patients who died had a medical history of malignancies during the previous 5 years (15). A systematic review and meta-analysis of 8 studies in predominantly Chinese populations $(n=46.248$, searches run 25 Feb $2020)$ found diabetes as with $8 \%(95 \% \mathrm{Cl} 6 \%-11 \%)$ as the second most prevalent comorbidity (after hypertension) in people hospitalized with COVID-19 (16). This was in line with another systematic review and meta-analysis of six studies ( $n=1527 ; 4$ studies included in the previous review) demonstrating that $9.7 \%(6.9 \%-12.5 \%)$ of COVID-19 infected patients had diabetes.

DPP4/CD26 is a ubiquitous membrane-bound aminopeptidase and has multiple physiological functions. These include the $T$ cell receptor-mediated $T$ cell activation and proliferation (17) as well as regulation of glucose homeostasis and its importance as highlighted by the approval of DPP4 inhibitors as established glucose-lowering therapy in type 2 diabetes (18). Modeling of the structure of the SARS-CoV-2 spike protein predicts an interaction of DPP4 in addition to ACE2 (19) (20). Interestingly, a correlation between DPP4 and ACE2 was found suggesting that both membrane proteins are relevant for the virus entry (21). DPP4 inhibitors are small molecules, which enter the catalytic pocket of the dimeric structure of DPP4 and bind to the catalytic site, thereby preventing the proteolytic activity (22). The coexpression of ACE2 and DPP4 as receptors of spike glycoproteins postulates that different human CoVs target similar cell types across different human tissues and explain the presence of comparable clinical features in patients infected with different CoVs. Furthermore, DPP4 acted as a CoV co-receptor suggesting a similar mechanism for the entry of SARS-CoV-2 (23). 
In this study, the coexpression of HT-SARS-CoV-2 and immune system process genes was evaluated followed by STRING analysis to determine the functional interactions between HT- SARS-CoV-2 and immune system genes. The STRING database network integrates direct and indirect/functional proteinprotein interactions, such as stable physical associations, transient binding, substrate chaining, information relay and others (24). Despite many limitations due to the low number of patients, the retrospective nature of evidence and the limited patients' follow-up, these data provide early insights into how the management of patients with cancer and/or diabetes mellitus might be affected by the COVID19 pandemic. This is an important issue, since cancer and diabetes mellitus are risk factors for disease progression and such patients were shown to present more severe symptoms and unfavorable outcomes upon COVID-19 infection (25-27).

\section{Materials And Methods}

\section{Gene ontology}

The Gene Ontology source (GO; http://geneontology.org) provides structured, computable knowledge regarding the functions and products of genes. In addition, GO enrichment analyses included three categories, biological process (BP), molecular function (MF) and cellular component (CC). For our studies, the GO term "immune system process (GO: 0002376)" was selected, which comprises 2811 genes.

\section{Networking analyses and drug screening}

The protein-protein network was constructed using the STRING Database (String v10) (28) as proteinprotein interaction network, which also analyze functional enrichments. STRING consisted of 24.6 million proteins with more than 2000 million interactions. The coexpression scores in STRING v10 are computed employing a revised and improved pipeline using high throughput microarray gene expression data deposited in NCBI GEO, mining of databases and literature as well as predictions based on genomic context analysis. The gene expression values are then correlated gene-by-gene (Pearson correlation) and the resulting values are calibrated against common membership in KEGG pathway maps to compute STRING scores.

To search for significant drug molecules and determine potential drug-associated mechanisms upon COVID-19 infections, the Search Tool for Interactions of Chemicals (STITCH, stitch.embl.de), a chemical protein interaction database that contains 0.5 million compounds and 9.6 million proteins, was employed.

\section{Samples and patients}

The R2 Genomics Platform is a web-based genomics analysis and visualization platform and allows biomedical researchers to integrate, analyze and visualize clinical and genomics data. Transcriptome data of healthy and cancer patients were sourced from The Cancer Genome Atlas (TCGA) dataset (http://cancergenome.nih.gov/) and microarray data of NCBI Gene Expression Omnibus (NCBI GEO) (29). 
The datasets were available on R2 Genomics Analysis and Visualization Platform (http://r2.amc.nl): (i) Normal Various - 353 samples (30), (ii) Mixed Cancer - 11003 samples (TCGA; https://www.cancer.gov/tcga), (iii) Pancreatic Islets - Normal - 87 samples (31), (iv) Pancreas - Mixed Tumor - 90 samples (32), (v) Pancreatic Islets - Diabetes - 128 samples (33).

\section{Correlation analysis}

Gene correlation analyses were performed with the R2: Genomics Analysis and Visualization Platform (http://r2.amc.nl); the genes and pathways associated with ANXA2 were tested by GO and KEGG pathway analysis. Correlation statistics were calculated using the R2 platform and $p<0.05$ was statistically significant.

\section{Statistical analysis}

Statistical computations and drawings of figures were performed with several packages (ggplot2 and circlize) in the statistical software environment R, version 3.3.2 (http://www.r- project.org).

\section{Results}

\section{Identification of protein associations}

The three human proteins ACE2, TMPRSS2 and FURIN used by SARS-CoV-2 to efficiently entry into human cells were studied for their functional protein associations using the STRING database. TMPRSS2 was networked as a center point associated with both ACE2 and FURIN, while ACE2 and FURIN showed no interactions between each other (Supplementary Figure 1). In order to study the immune system interactions of the human targets, the GO term immune system process' (GO: 0002376) from the Gene Ontology resource was analyzed. In total, 2811 genes (Supplementary Table 1) were studied for functional protein association networks using STRING (string-db.org). This analysis produced 24072 protein associations (Supplementary Table 2), among which ACE2 shared 21 protein associations (Figure 1A), while TMPRSS2 shared 17 protein associations (Figure 1B). With 64 proteins, FURIN indicated the highest associations (Figure 1C). Among the protein associations within the three HT-SARS-CoV-2, DPP4 shared associations with ACE2, TMPRSS2 and FURIN (Figure 2), while ADAM17, glycoprotein 2 (GP2), transferrin receptor 1 (TFGC) were the common network elements with ACE2 and FURIN. CTSL, GAPDH, MYC, AKT1 and SRC were functionally associated with TMPRSS2 and FURIN (Table 1).

Although no genes were co-expressed at significant levels $(p \leq 0.05)$ (Table 1), DPP4 was co-expressed with the three HT-SARS-CoV-2 analyzed with a p-value of 0.06. Likewise, FURIN with ADAM17 ( 0.063 ) and MYC ( $p$ 0.061) and CTSL with TMPRSS2 ( $p$ 0.061) and FURIN ( $p 0.062)$ shared coexpression patterns. The top text-mining score was obtained by FURIN/GP2 (0.859) followed by ACE2/DPP4 (0.717) and ACE2/ADAM17 (0.706). On the other hand, ACE2/DPP4 had the highest STRING score (0.942) followed by FURIN/GP2 and (0.859) and ACE2/ADAM17 (0.707) (Table 1).

\section{Determination of genes correlating with HT-SARS-CoV-2 in cancer}


The ACE2, TMPRSS2 and FURIN expression was correlated to every single gene in the five different datasets described in Materials and Methods. The top 1500 genes of the respective association i.e., having a highly significant correlation $(p<0.01)$ in the corresponding dataset, are summarized in Supplementary Table 3. From the common STRING associations between our human target genes, 19 associations of 8 commonly networking genes were plotted to the 3 HT-SARS-CoV-2 (Table 2) in mixed healthy (323 samples) and cancer (11003 samples) tissues. Such collected data allowed a classification in four distinct segments: (i) associations between human target genes, (ii) triple association of DPP4 and (iii) double associations of FURIN with either ACE2 or (iv) with TMPRSS2, respectively. The top segment of Table 2 represents the positive correlations involving TMPRSS2 in both healthy and tumor datasets. The central association marker, DPP4 positively correlates with ACE2 (normal: R 0.308; cancers: 0.382), TMPRSS2 (normal: R 0.524; cancers: 0.382) and FURIN (normal: R 0.498; cancers: R 0.246) in both datasets (Figure 3). ADAM17 exhibits negative correlations with ACE2 (normal: R -0.187; cancers: R 0.044) and FURIN (normal: R -0.281; cancers: R -0.079). GP2 and TFRC showed differential association patterns with ACE2 and FURIN. The common TMPRSS2 and FURIN associated genes, CTSL, MYC, AKT1 and SRC, displayed positive correlations with their corresponding subjects except for FURIN and MYC correlation in cancers (R 0.012; $p$ 0.199). TMPRSS2 negatively correlated with GAPDH (Table 2).

To explore gene association patterns in the wide range of datasets, ACE2, TMPRSS2 and FURIN were correlated to DPP4, SRC and ADAM17 expression regardless of the tissue and the disease. ACE2 and TMPRSS2 displayed a positive correlation, whereas FURIN showed a negative correlation with their counterparts (Table 3 and Figure 3).

\section{Correlation pattern of SARS-CoV-2 targets in pancreatic tissues}

Healthy pancreatic tissues exhibit distinct associations when compared to pancreatic cancer tissues or tissue samples from patients suffering from diabetes mellitus. The HT-SARS-CoV2 ACE2 and TMPRSS2 were positively associated with healthy pancreatic tissue, while a non-significant positive association was noted in the context of cancer and diabetes mellitus. A positive correlation between TMPRSS2 and FURIN was found in the diseased, but not in the healthy pancreas (Table 3). Interestingly, ACE2 and DPP4 were connected irrespective of the pancreatic nature. DPP4 was differentially expressed with TMPRSS2 and FURIN; TMPRSS2 was positively expressed in healthy and negatively expressed in the diseased pancreas.

FURIN expression was not correlated with DPP4 except for pancreatic cancer, where a negative correlation was found. The FURIN/ADAM17 axis remained negatively associated with pancreatic tissues. Interestingly, TMPRSS2 positively correlated with SRC in the pancreatic tissues in mixed healthy and cancer datasets. Other double associating genes lack any specific correlation pattern. FURIN and MYC were positively associated with healthy tissues and pancreatic cancer. However, FURIN acted positively with MYC in pancreatic cancer, but not in the mixed cancer dataset. AKT1 with TMPRSS2 and FURIN were positively correlated in all contexts except for pancreatic cancer and healthy pancreas, respectively. 
Interestingly, many networking genes were not correlated in pancreatic tissues. These include ACE2/ADAM17, FURIN/GAPDH, ACE2/FURIN, ACE2/GP2 and ACE2/TFRC, respectively.

\section{Identification of drug candidates for HT-SARS-CoV-2 and co-expressed genes}

A total of 16 target drugs (Table 4) and 32 drug-associated genes (Supplementary Table 4) were acquired from STITCH analysis. As shown in Figure 3, ACE2/DPP4 and FURIN/ADAM17 were predicted to have drug connections, whereas TMPRSS2/SRC had no predicted drug connection. Several inhibitors of the angiotensin receptor (ACE) and DPP4, such as sitagliptin, teneligliptin, linagliptin, vildagliptin and saxagliptin, were identified. For FURIN and ADAM17 therapeutic involvement via calcium ions and Zn (II and in addition for ADAM17, an involvement via IK-682 were identified.

\section{Discussion}

In this comprehensive study, the critical immune-related associations with the HT-SARS- CoV-2 ACE2, TMPRSS2 and FURIN were identified using bioinformatics analyses. SARS- CoV-2 requires the two human proteins ACE2 and TMPRSS2 for cell entry (6), which are the same proteins used by SARS-CoV (34), the cause of the 2002-2004 SARS epidemic. The SARS-CoV-2 viral genome encodes for 29 different proteins. A recent study by Gordon and co-workers expressed and purified 26 of these proteins and analyzed them for protein- protein interactions (35). In total, 332 high confident protein-protein interactions between SARS-CoV-2 and human proteins and 66 druggable human proteins were identified. Interestingly, two sets of pharmacological drugs had antiviral activities. These included inhibitors of mRNA translation of two sigma receptors.

FURIN and ADAM17 are both intertwined with Notch, an evolutionarily conserved system of communication between adjacent cells, and thus targeting of Notch could represent an alternative approach to inhibit FURIN and upregulate ADAM17 (36). In this context, it is noteworthy that ADAM17 is involved in the shedding of ACE2 (37). With this approach, it is impossible to identify host proteins crucial for viral infection, which do not interact directly with viral proteins, as is the case for FURIN and ADAM17. Thus, hypothesis-driven studies based on the knowledge of the molecular details of virus-cell interaction are still crucial for the identification of therapeutic targets to treat COVID-19.

Studies have shown a strong correlation between the severity of the disease and concentrations of cytokines, such as IL2, IL7, IL10, GCSF, MCP1 and TNF-alpha (38). Notch and IL-6 cooperate to activate the immune system and perpetuate the cytokine storm. In macrophages, DII4/Notch signaling promotes the production of inflammatory cytokines, including IL-6 (36). IL-6, in turn, increases the expression of the Notch ligands (DII 1,4) thus amplifying the Notch signaling and establishing a feedback loop that promotes the further production of IL-6. In T cells, Notch signaling triggered by DII1/DII4 ligands promotes inflammatory Th1/Th17 cytokines, while Jagged1 ligands dampen the IL-6-induced Th17 activation (36).So far, there exist only a few reports on the specific mechanisms of interaction networks and associations of TMPRSS2, ACE2 and FURIN with their targets. Using bioinformatics, we here show that ACE2 may participate in the Notch signaling to exert immune effects. However, the relationship 
between these molecules has to be analyzed in more detail concerning possible targeting of ACE2/DPP4 and FURIN/ADAM17-related factors in diseased COVID-19 patients.

Angiotensin receptor blockers (ARBs) comparable to angiotensin converting enzyme (ACE) inhibitors, which act by preventing the formation of angiotensin II rather than by blocking the binding of angiotensin II to muscles on blood vessels. The therapeutic indication for ARBs prescription comprises control of high blood pressure, treating heart failure and preventing kidney failure, especially in patients suffering from diabetes mellitus. Since ACE inhibitors are associated with cough, accumulation of bradykinin and angioedema, ARBs, like losartan and captopril, might be a more favorable therapeutic approach by blocking the binding and attachment of SARS-CoV-2 RBD to ACE2-expressing cells, thus inhibiting the infection of host cells (39). Ferrario and colleagues reported that the administration of either ACE inhibitors or ARBs increased the levels of ACE2 mRNA in Lewis rats compared to rats receiving placebo (40). In particular, cardiac levels of ACE2 mRNA expression increased by 4.7-fold or 2.8-fold in rats treated with losartan, which was accompanied by increased ACE2 activity. Treatment with the ACE inhibitor captopril can significantly increase ACE2 protein expression in rats with acute lung injury (41). Furthermore, Mourad and Levy suggested aliskiren treatment as an option in the context of SARS-CoV-2 infection, since it can reduce the expression of ACE2 (42).

Early trials in the 1990s showed that DPP4 inhibitors improve glycemia in animals. Subsequent clinical studies during the 2000s showed a glucose-lowering authority of DPP4 inhibitors in humans with type 2 diabetes as monotherapy or as combination with other therapies, i.e., metformin, sulfonylureas, tiazolidinediones or exogenous insulin. Five DPP-4 inhibitors (sitagliptin, vildagliptin, alogliptin, saxagliptin and linagliptin) were approved by regulatory authorities for treatment of type 2 diabetes and entered the market between 2006 and 2013 (22).

\section{Conclusions}

The results of the present study suggest novel drug candidates for COVID-19 infections and underscore that new treatment options can be postulated by using different bioinformatics tools, which now have to be experimentally proven.

\section{Abbreviations}

ACE-2, angiotensin converting enzyme II; ADAM17, ADAM metallopeptidase domain 17; BP, biological process; CC, cellular component; CoV, coronavirus; DPP4, dipeptidyl peptidase-4; GO, gene ontology; GP2, glycoprotein 2; HT-SARS-CoV-2, human targets of SARS-CoV-2; MF, molecular function; nCoV, novel coronavirus; TFRC, transferrin receptor 1; TMPRSS2, transmembrane serine protease 2

\section{Declarations}

\section{Ethics approval and consent to participate}


not applicable

\section{Consent for publication}

All authors read the final version of this article and gave consent for publication.

\section{Availability of data and materials}

The data used to support the findings of this study are included within the article and are public available.

\section{Competing interests}

There exist no competing interests of all authors.

\section{Funding}

The work was supported by a grant from the Wilhelm-Sander-Stiftung (No: 2019.076.1).

\section{Authors' contributions}

Karthikeyan Subbarayan and Barbara Seliger designed the project. Karthikeyan Subbarayan and Kamatchi Ulagappan performed the bioinformatics and analyses. Claudia Wickenhauser, Karthikeyan Subbarayan and Barbara Seliger interpreted the data and wrote the paper.

\section{Acknowledgements}

We would like to thank N. Ott and V. Goldhorn for excellent secretarial help.

\section{References}

1. Cui J, Li F, Shi ZL. Origin and evolution of pathogenic coronaviruses. Nat Rev Microbiol. 2019;17(3):181-92.

2. Wu A, Peng Y, Huang B, Ding X, Wang X, Niu P, et al. Genome Composition and Divergence of the Novel Coronavirus (2019-nCoV) Originating in China. Cell Host Microbe. 2020;27(3):325-8.

3. Zhou P, Yang XL, Wang XG, Hu B, Zhang L, Zhang W, et al. A pneumonia outbreak associated with a new coronavirus of probable bat origin. Nature. 2020;579(7798):270-3.

4. Letko M, Marzi A, Munster V. Functional assessment of cell entry and receptor usage for SARS-CoV-2 and other lineage B betacoronaviruses. Nat Microbiol. 2020;5(4):562-9.

5. Lu G, Hu Y, Wang Q, Qi J, Gao F, Li Y, et al. Molecular basis of binding between novel human coronavirus MERS-CoV and its receptor CD26. Nature. 2013;500(7461):227- 31.

6. Hoffmann M, Kleine-Weber H, Schroeder S, Kruger N, Herrler T, Erichsen S, et SARS-CoV-2 Cell Entry Depends on ACE2 and TMPRSS2 and Is Blocked by a Clinically Proven Protease Inhibitor. Cell. 2020;181(2):271-80 e8. 
7. Wrapp D, Wang N, Corbett KS, Goldsmith JA, Hsieh CL, Abiona O, et al. Cryo-EM structure of the 2019nCoV spike in the prefusion conformation. Science. 2020;367(6483):1260-3.

8. Lukassen S, Chua RL, Trefzer T, Kahn NC, Schneider MA, Muley T, et al. SARS- CoV-2 receptor ACE2 and TMPRSS2 are primarily expressed in bronchial transient secretory cells. EMBO J. 2020;39(10):e105114.

9. Zhang YZ, Holmes EC. A Genomic Perspective on the Origin and Emergence of SARS-CoV-2. Cell. 2020;181(2):223-7.

10. Tay MZ, Poh CM, Renia L, MacAry PA, Ng LFP. The trinity of COVID-19: immunity, inflammation and intervention. Nat Rev Immunol. 2020;20(6):363-74.

11. Blanco-Melo D, Nilsson-Payant BE, Liu WC, Uhl S, Hoagland D, Moller R, et Imbalanced Host Response to SARS-CoV-2 Drives Development of COVID-19. Cell. 2020;181(5):1036-45 e9.

12. Mehta P, McAuley DF, Brown M, Sanchez E, Tattersall RS, Manson JJ, et al. COVID- 19: consider cytokine storm syndromes and immunosuppression. Lancet. 2020;395(10229):1033-4.

13. Dariya B, Nagaraju GP. Understanding novel COVID-19: Its impact on organ failure and risk assessment for diabetic and cancer patients. Cytokine Growth Factor Rev. 2020;53:43-52.

14. Franceschini A, Szklarczyk D, Frankild S, Kuhn M, Simonovic M, Roth A, et STRING v9.1: proteinprotein interaction networks, with increased coverage and integration. Nucleic Acids Res. 2013;41(Database issue):D808-15.

15. Onder G, Rezza G, Brusaferro S. Case-Fatality Rate and Characteristics of Patients Dying in Relation to COVID-19 in Italy. JAM

16. Yang J, Zheng Y, Gou X, Pu K, Chen Z, Guo Q, et al. Prevalence of comorbidities and its effects in patients infected with SARS-CoV-2: a systematic review and meta-analysis. Int J Infect Dis. 2020;94:91-5.

17. Radzikowska U, Ding M, Tan G, Zhakparov D, Peng Y, Wawrzyniak P, et Distribution of ACE2, CD147, CD26 and other SARS-CoV-2 associated molecules in tissues and immune cells in health and in asthma, COPD, obesity, hypertension, and COVID-19 risk factors. Allergy. 2020.

18. Kirby M, Yu DM, O'Connor S, Gorrell Inhibitor selectivity in the clinical application of dipeptidyl peptidase-4 inhibition. Clin Sci (Lond). 2009;118(1):31-41.

19. Bassendine MF, Bridge SH, McCaughan GW, Gorrell MD. COVID-19 and comorbidities: A role for dipeptidyl peptidase 4 (DPP4) in disease severity? J Diabetes.

20. Vankadari N, Wilce JA. Emerging WuHan (COVID-19) coronavirus: glycan shield and structure prediction of spike glycoprotein and its interaction with human CD26. Emerg Microbes Infect. 2020;9(1):601-4.

21. Qi F, Qian S, Zhang S, Zhang Z. Single cell RNA sequencing of 13 human tissues identify cell types and receptors of human coronaviruses. Biochem Biophys Res Commun. 2020;526(1):135-40.

22. Ahren B. DPP-4 Inhibition and the Path to Clinical Proof. Front Endocrinol (Lausanne). 2019;10:376. 
23. Raj VS, Mou H, Smits SL, Dekkers DH, Muller MA, Dijkman R, et al. Dipeptidyl peptidase 4 is a functional receptor for the emerging human coronavirus-EMC. 2013;495(7440):251-4.

24. Szklarczyk D, Franceschini A, Wyder S, Forslund K, Heller D, Huerta-Cepas J, et STRING v10: proteinprotein interaction networks, integrated over the tree of life. Nucleic Acids Res. 2015;43(Database issue):D447-52.

25. Sidaway P. COVID-19 and cancer: what we know so far. Nat Rev Clin 2020;17(6):336.

26. Joensen LE, Madsen KP, Holm L, Nielsen KA, Rod MH, Petersen AA, et al. Diabetes and COVID-19: psychosocial consequences of the COVID-19 pandemic in people with diabetes in Denmark-what characterizes people with high levels of COVID-19-related worries? Diabet Med. 2020;37(7):1146-54.

27. Ye L, Xu J, Zhang T, Lin X, Pan X, Zeng W, et al. Global Burden of Non- communicable Diseases Attributable to High Fasting Plasma Glucose. J Diabetes.

28. Szklarczyk D, Gable AL, Lyon D, Junge A, Wyder S, Huerta-Cepas J, et al. STRING v11: protein-protein association networks with increased coverage, supporting functional discovery in genome-wide experimental datasets. Nucleic Acids Res. 2019;47(D1):D607- D13.

29. Barrett T, Wilhite SE, Ledoux P, Evangelista C, Kim IF, Tomashevsky M, et al. NCBI GEO: archive for functional genomics data sets-update. Nucleic Acids Res. 2013;41(Database issue):D991-5.

30. Roth RB, Hevezi P, Lee J, Willhite D, Lechner SM, Foster AC, et al. Gene expression analyses reveal molecular relationships among 20 regions of the human CNS. Neurogenetics. 2006;7(2):67-80.

31. Hall E, Volkov P, Dayeh T, Esguerra JL, Salo S, Eliasson L, et al. Sex differences in the genome-wide DNA methylation pattern and impact on gene expression, microRNA levels and insulin secretion in human pancreatic islets. Genome Biol. 2014;15(12):522.

32. Zhang G, He P, Tan H, Budhu A, Gaedcke J, Ghadimi BM, et al. Integration of metabolomics and transcriptomics revealed a fatty acid network exerting growth inhibitory effects in human pancreatic cancer. Clin Cancer Res. 2013;19(18):4983-93.

33. Krus U, King BC, Nagaraj V, Gandasi NR, Sjolander J, Buda P, et al. The complement inhibitor CD59 regulates insulin secretion by modulating exocytotic events. Cell Metab. 2014;19(5):883-90.

34. Shulla A, Heald-Sargent T, Subramanya G, Zhao J, Perlman S, Gallagher T. A transmembrane serine protease is linked to the severe acute respiratory syndrome coronavirus receptor and activates virus entry. J Virol. 2011;85(2):873-82.

35. Gordon DE, Jang GM, Bouhaddou M, Xu J, Obernier K, White KM, et al. A SARS- CoV-2 protein interaction map reveals targets for drug repurposing. Nature. 2020;583(7816):459-68.

36. Rizzo P, Vieceli Dalla Sega F, Fortini F, Marracino L, Rapezzi C, Ferrari R. COVID-19 in the heart and the lungs: could we "Notch" the inflammatory storm? Basic Res Cardiol. 2020;115(3):31.

37. Lambert DW, Yarski M, Warner FJ, Thornhill P, Parkin ET, Smith Al, et al. Tumor necrosis factor-alpha convertase (ADAM17) mediates regulated ectodomain shedding of the severe-acute respiratory syndrome-coronavirus (SARS-CoV) receptor, angiotensin- converting enzyme-2 (ACE2). J Biol Chem. 2005;280(34):30113-9. 
38. Singhal T. A Review of Coronavirus Disease-2019 (COVID-19). Indian J 2020;87(4):281-6.

39. Sanchis-Gomar F, Lavie CJ, Perez-Quilis C, Henry BM, Lippi G. Angiotensin- Converting Enzyme 2 and Antihypertensives (Angiotensin Receptor Blockers and Angiotensin-Converting Enzyme Inhibitors) in Coronavirus Disease 2019. Mayo Clin Proc. 2020;95(6):1222-30.

40. Ferrario CM, Jessup J, Chappell MC, Averill DB, Brosnihan KB, Tallant EA, et Effect of angiotensinconverting enzyme inhibition and angiotensin II receptor blockers on cardiac angiotensin-converting enzyme 2. Circulation. 2005;111(20):2605-10.

41. Li Y, Zeng Z, Li Y, Huang W, Zhou M, Zhang X, et al. Angiotensin-converting enzyme inhibition attenuates lipopolysaccharide-induced lung injury by regulating the balance between angiotensinconverting enzyme and angiotensin-converting enzyme 2 and inhibiting mitogen-activated protein kinase activation. Shock. 2015;43(4):395-404.

42. Mourad JJ, Levy BI. Interaction between RAAS inhibitors and ACE2 in the context of COVID-19. Nat Rev Cardiol. 2020;17(5):313.

\section{Tables}

Table 1: Identification of immune process relevant genes associated with three HT-SARS- CoV-2 


\begin{tabular}{|c|c|c|c|c|}
\hline \multirow{2}{*}{ gene $\mathbf{A}$} & \multirow{2}{*}{ gene B } & \multicolumn{3}{|c|}{ STRING } \\
\hline & & coexpression & text mining & score \\
\hline \multicolumn{5}{|c|}{ associations with human targets of SARS-CoV-2 } \\
\hline ACE2 & TMPRSS2 & 0.099 & 0.39 & 0.427 \\
\hline TMPRSS2 & FURIN & 0 & 0.531 & 0.531 \\
\hline \multicolumn{5}{|c|}{ triple associations of DPP4 } \\
\hline ACE2 & DPP4 & 0.061 & 0.717 & 0.942 \\
\hline TMPRSS2 & DPP4 & 0.062 & 0.396 & 0.409 \\
\hline FURIN & DPP4 & 0.061 & 0.424 & 0.439 \\
\hline \multicolumn{5}{|c|}{ double associations with ACE2 and FURIN } \\
\hline ACE2 & ADAM17 & 0 & 0.706 & 0.707 \\
\hline FURIN & ADAM17 & 0.063 & 0.619 & 0.628 \\
\hline ACE2 & GP2 & 0 & 0.571 & 0.571 \\
\hline FURIN & GP2 & 0 & 0.859 & 0.859 \\
\hline ACE2 & TFRC & 0 & 0.663 & 0.663 \\
\hline FURIN & TFRC & 0.063 & 0.498 & 0.514 \\
\hline \multicolumn{5}{|c|}{ double associations with TMPRSS2 and FURIN } \\
\hline TMPRSS2 & CTSL & 0.061 & 0.469 & 0.48 \\
\hline FURIN & CTSL & 0.062 & 0.49 & 0.514 \\
\hline TMPRSS2 & MYC & 0 & 0.518 & 0.518 \\
\hline FURIN & MYC & 0.061 & 0.415 & 0.427 \\
\hline TMPRSS2 & AKT1 & 0 & 0.472 & 0.476 \\
\hline FURIN & AKT1 & 0.106 & 0.429 & 0.479 \\
\hline TMPRSS2 & SRC & 0 & 0.4 & 0.405 \\
\hline FURIN & $\mathrm{SRC}$ & 0.095 & 0.6 & 0.631 \\
\hline TMPRSS2 & GAPDH & 0 & 0.425 & 0.448 \\
\hline FURIN & GAPDH & 0 & 0.414 & 0.414 \\
\hline
\end{tabular}

The association of HT-SARS-Cov-2 with immune relevant genes was determined by STRING analyses and represented as co-expression, text mining and score.

Table 2: Association of HT-SARS-CoV-2 with immune process relevant genes in mixed cancers vs. mixed normal tissues 


\begin{tabular}{|c|c|c|c|c|c|}
\hline \multirow[b]{2}{*}{ Gene A } & \multirow[b]{2}{*}{ Gene B } & \multicolumn{2}{|c|}{ Mixed-Normal } & \multicolumn{2}{|c|}{ Mixed-Cancers } \\
\hline & & $\mathbf{R}$ & $P$ & $\mathbf{R}$ & $P$ \\
\hline \multicolumn{6}{|c|}{ Assciations with human targets of SARS-CoV-2 } \\
\hline ACE2 & TMPRSS2 & 0.29 & $8.63 \mathrm{E}-07$ & 0.164 & $1.42 \mathrm{E}-67$ \\
\hline TMPRSS2 & FURIN & 0.376 & $2.78 \mathrm{E}-12$ & 0.29 & $1.47 \mathrm{E}-211$ \\
\hline \multicolumn{6}{|c|}{ Triple assciations of DPP4 } \\
\hline ACE2 & DPP4 & 0.308 & $1.66 \mathrm{E}-07$ & 0.382 & $0.00 \mathrm{E}+00$ \\
\hline TMPRSS2 & DPP4 & 0.524 & $4.05 \mathrm{E}-24$ & 0.382 & $0.00 \mathrm{E}+00$ \\
\hline FURIN & DPP4 & 0.498 & $6.96 \mathrm{E}-20$ & 0.246 & $1.82 \mathrm{E}-150$ \\
\hline \multicolumn{6}{|c|}{ Double associations with ACE2 and FURIN } \\
\hline ACE2 & ADAM17 & -0.187 & $4.22 \mathrm{E}-04$ & -0.044 & $3.76 \mathrm{E}-06$ \\
\hline FURIN & ADAM17 & -0.281 & 7.96E-07 & -0.079 & $7.59 \mathrm{E}-17$ \\
\hline ACE2 & GP2 & 0.108 & 0.043 & -0.045 & 2.64E-06 \\
\hline FURIN & GP2 & 0.006 & 0.905 & 0.098 & 8.87E-25 \\
\hline ACE2 & TFRC & 0.085 & 0.11 & 0.085 & 4.23E-19 \\
\hline FURIN & TFRC & 0.057 & 0.286 & 0.05 & $1.61 \mathrm{E}-07$ \\
\hline \multicolumn{6}{|c|}{ Double associations with TMPRSS2 and FURIN } \\
\hline TMPRSS2 & CTSL & 0.111 & 0.038 & 0.129 & $3.93 \mathrm{E}-42$ \\
\hline FURIN & CTSL & 0.154 & 3.83E-03 & 0.175 & $1.44 \mathrm{E}-76$ \\
\hline TMPRSS2 & MYC & 0.274 & $1.74 \mathrm{E}-07$ & 0.022 & $2.20 \mathrm{E}-02$ \\
\hline FURIN & MYC & 0.149 & $5.04 \mathrm{E}-03$ & 0.012 & 0.199 \\
\hline TMPRSS2 & AKT1 & 0.274 & $1.71 \mathrm{E}-07$ & 0.137 & $3.43 \mathrm{E}-47$ \\
\hline FURIN & AKT1 & 0.193 & 2.67E-04 & 0.21 & 2.30E-109 \\
\hline TMPRSS2 & SRC & 0.288 & 3.75E-08 & 0.309 & $1.86 \mathrm{E}-240$ \\
\hline FURIN & SRC & 0.161 & $2.42 \mathrm{E}-03$ & 0.084 & $1.51 \mathrm{E}-18$ \\
\hline TMPRSS2 & GAPDH & -0.308 & 3.28E-09 & -0.314 & 7.39E-250 \\
\hline FURIN & GAPDH & -0.198 & 1.87E-04 & 0.023 & $1.80 \mathrm{E}-02$ \\
\hline
\end{tabular}

Correlation of HT-SARS-Cov2 with immune process relevant genes was performed by RZ2 genomics using mixed healthy and mixed cancer data sets and presented as correlation values (R) including the statistical significance $(\mathrm{P})$.

Table 3: Associations of HT-SARS-CoV-2 with immune process relevant genes in normal pancreas, pancreatic cancer and diabetic pancreatic tissues 


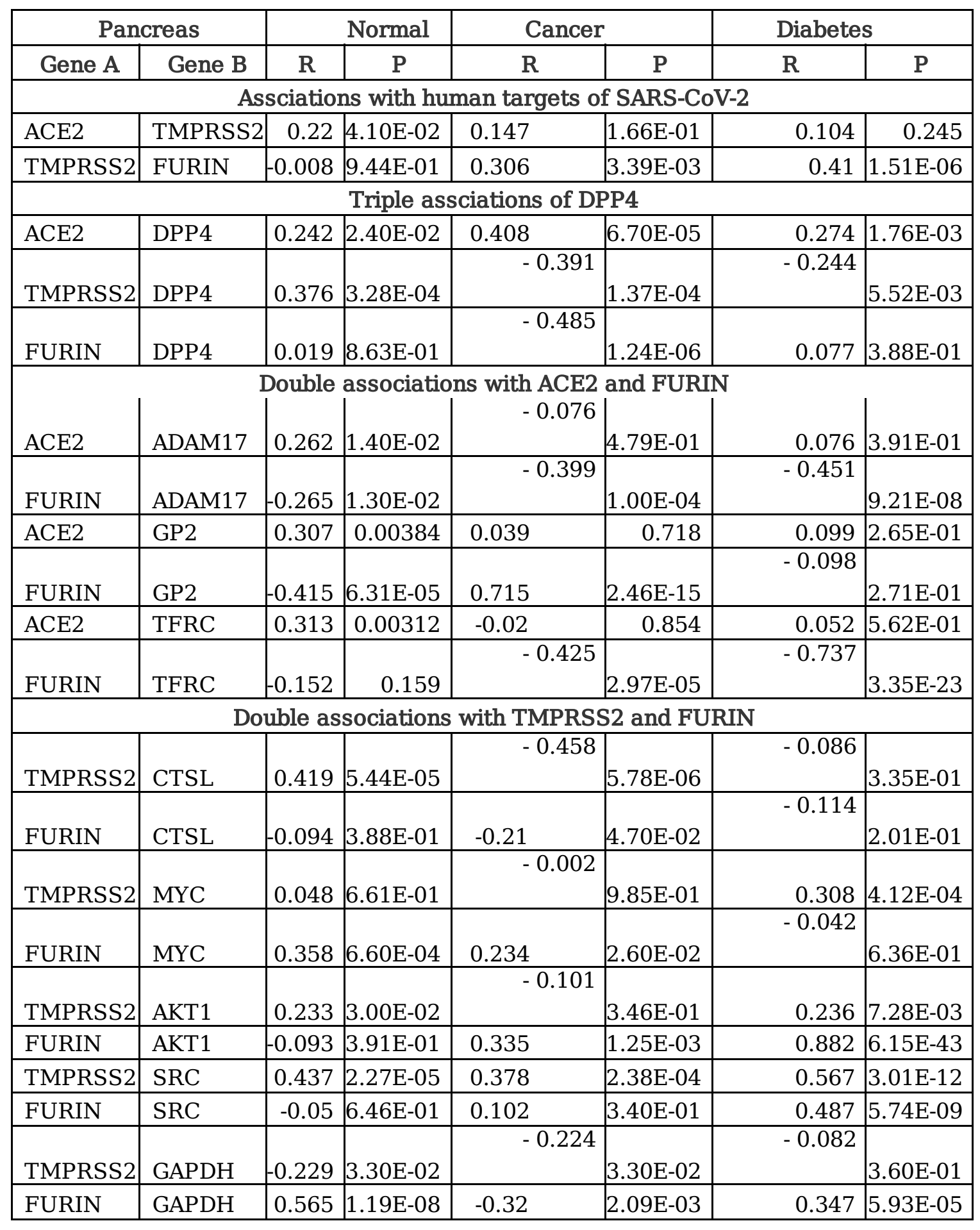

Correlation of HT-SARS-CoV-2 with immune process relevant genes was performed by R2 genomics using data sets obtained fromnormal pancreatic, pancreatic cancer and diabetic pancreatic tissues. Data are presented as correlation value $(R)$ including the statistical significance $(P)$.

Table 4: Known drug candidates targeting HT-SARS-CoV-2 and associated genes 


\begin{tabular}{|c|c|c|c|c|c|}
\hline gene & drug & $\begin{array}{c}\text { exp. } \\
\text { determined } \\
\text { interaction }\end{array}$ & $\begin{array}{l}\text { database } \\
\text { annotated }\end{array}$ & $\begin{array}{l}\text { automated text } \\
\text { mining }\end{array}$ & $\begin{array}{c}\text { combined } \\
\text { score }\end{array}$ \\
\hline ACE2 & losartan & 0 & 0.900 & 0.907 & 0.990 \\
\hline ACE2 & \begin{tabular}{|l|} 
captopril \\
\end{tabular} & 0.790 & 0.900 & 0.515 & 0.989 \\
\hline DPP4 & \begin{tabular}{|l|} 
sitagliptin \\
\end{tabular} & 0.900 & 0.800 & 0.968 & 0.999 \\
\hline DPP4 & \begin{tabular}{|l|l|} 
teneligliptin \\
\end{tabular} & 0.972 & 0.800 & 0.865 & 0.999 \\
\hline DPP4 & \begin{tabular}{|l|} 
linagliptin \\
\end{tabular} & 0.937 & 0.800 & 0.920 & 0.998 \\
\hline DPP4 & NVP-DPP728 & 0.915 & 0.800 & 0.893 & 0.998 \\
\hline DPP4 & \begin{tabular}{|l} 
vildagliptin \\
\end{tabular} & 0.797 & 0.800 & 0.932 & 0.997 \\
\hline DPP4 & \begin{tabular}{|l|} 
saxagliptin \\
\end{tabular} & 0.807 & 0.800 & 0.933 & 0.997 \\
\hline DPP4 & \begin{tabular}{|l} 
DB08743 \\
\end{tabular} & 0.957 & 0.800 & 0 & 0.991 \\
\hline DPP4 & CHEMBL445437 & 0.989 & 0 & 0 & 0.989 \\
\hline DPP4 & Pro-boroPro & 0.912 & 0.800 & 0.395 & 0.988 \\
\hline FURIN & calcium ions & 0.886 & 0.900 & 0.133 & 0.989 \\
\hline URIN & Zn(II & 0.498 & 0 & 0.120 & 0.545 \\
\hline ADAM17 & IK-682 & 0.978 & 0.800 & 0.169 & 0.996 \\
\hline ADAM17 & Zn(II & 0.800 & 0.900 & 0.139 & 0.981 \\
\hline ADAM17 & \begin{tabular}{|l|l} 
calcium ions \\
\end{tabular} & 0.800 & 0 & 0 & 0.800 \\
\hline
\end{tabular}

Drug candidates of HT-SARS-CoV-2 and immune process relevant genes were determined by STRING analysis and experimental interaction, database annotation, automatic text mining presented. In addition, the data were summarized in a combined score.

\section{Figures}


A

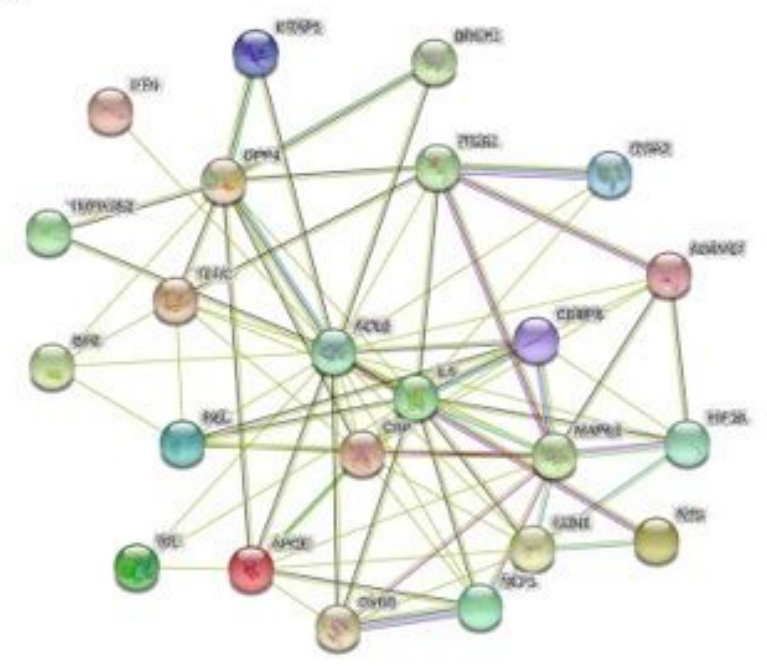

B

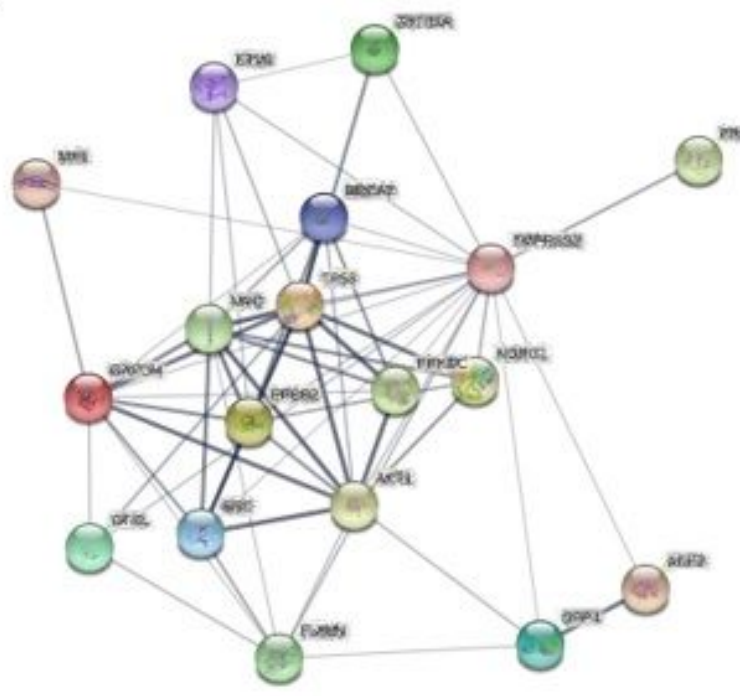

C

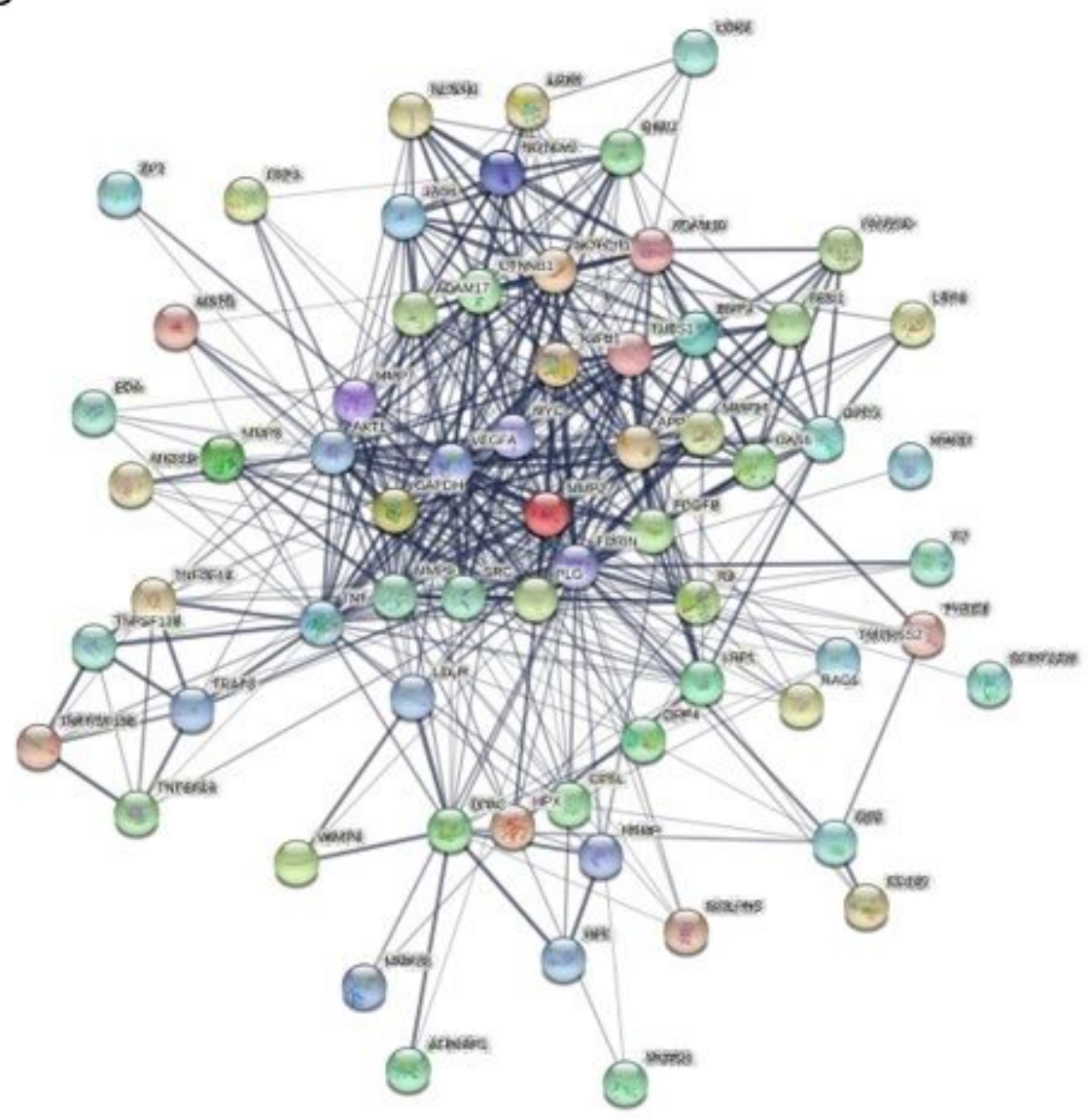

Figure 1

Construction and analysis of a protein-protein interaction network of HT-SARS-CoV-2 and immune genes. The protein-protein interaction network was visualized by STRING. The color saturation of the edges represents the confidence score of a functional association. A. ACE2; B. TMPRSS2; C. FURIN 


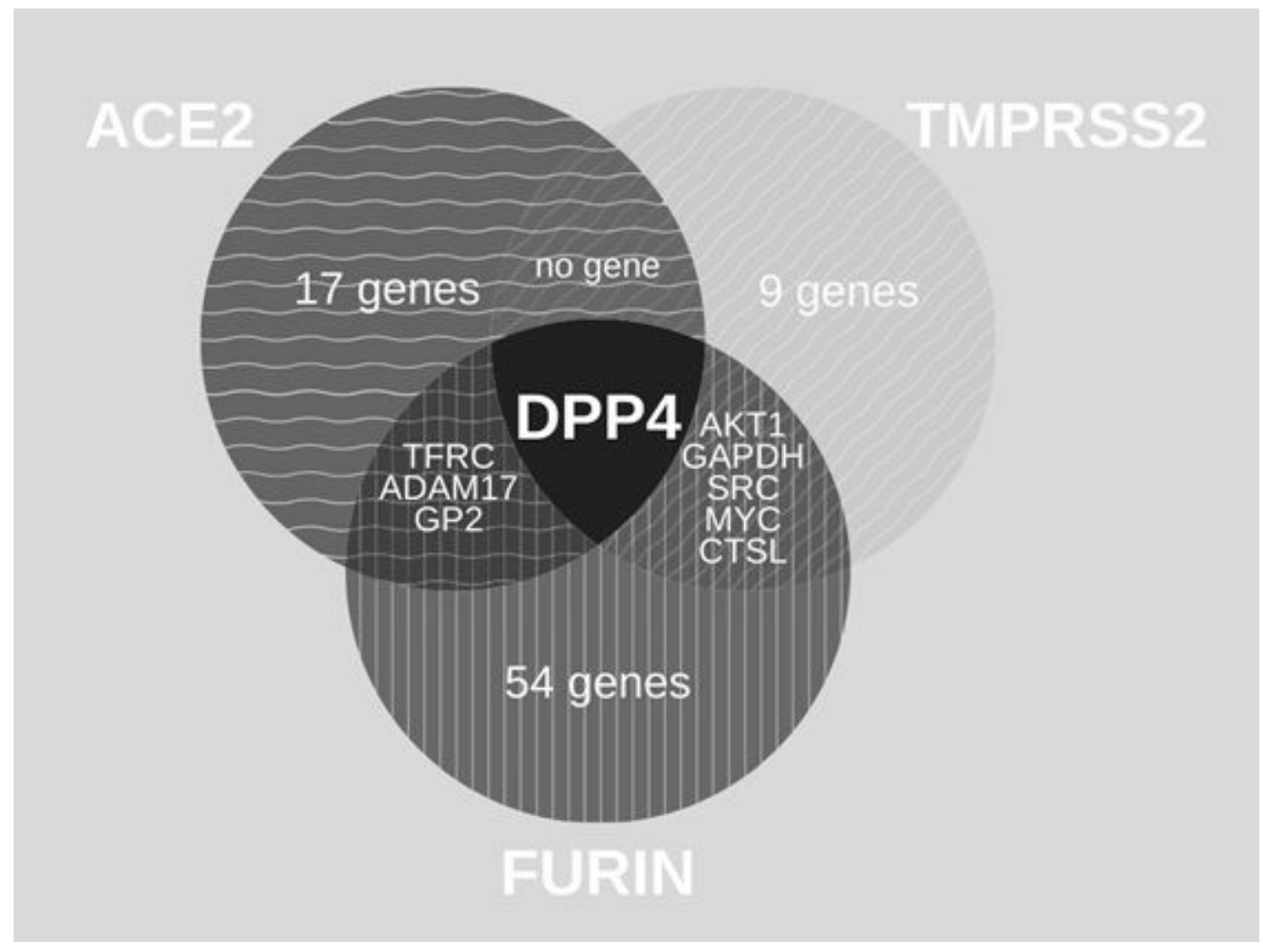

Figure 2

Venn diagrams of protein associations between HT-SARS-CoV-2 and immune genes. The association between the HT-SARS-CV-2 ACE2, TMPRSS2 and FURIN and 2811 immune relevant genes is demonstrated showing only one common immune molecule associated with all three HT-SARS-CoV-2. 

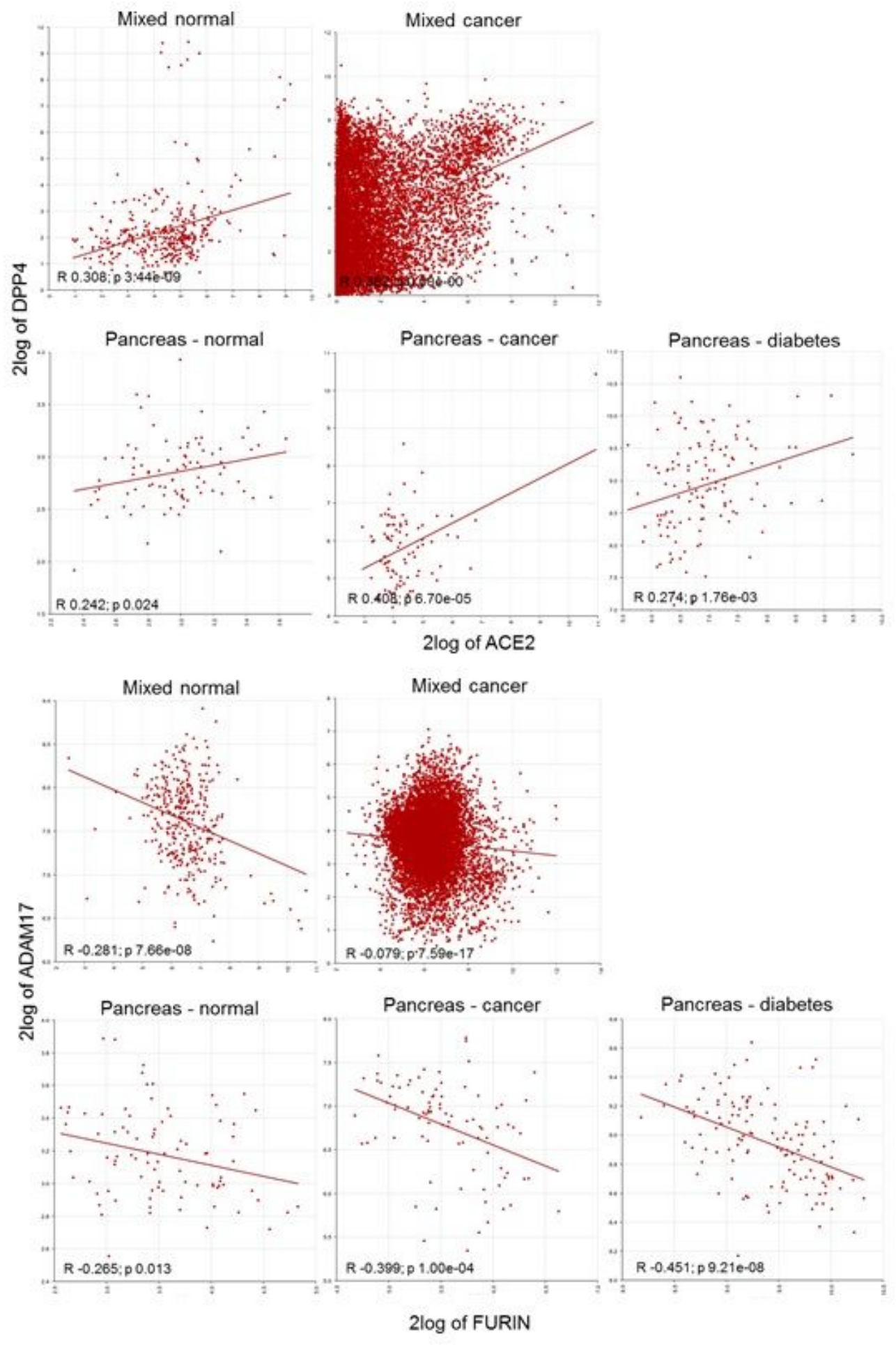

\section{Figure 3}

Correlation plot of HT-SARS-CoV-2 genes in mixed normal and cancers, and pancreatic normal, cancer and diabetic tissues. A. positive correlations of ACE2/DPP4; B. negative correlations of FURIN/ADAM17 

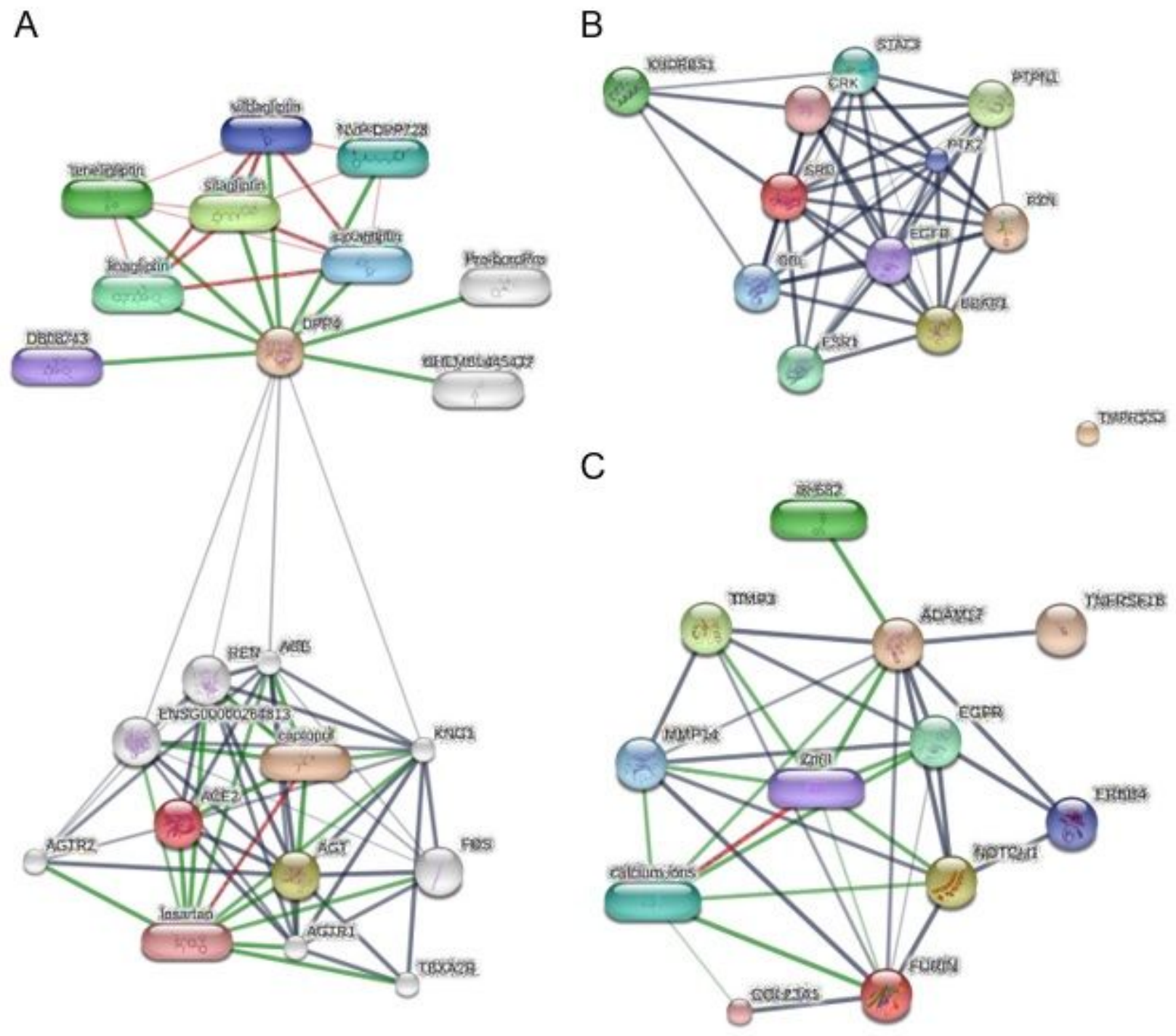

\section{Figure 4}

Drug-target networks of HT-SARS-CoV-2 using the STITCH database. The druggable targets of the association of HT-SARS-CoV-2 with immune process genes were curated from the STITCH database. Pillshaped and spheres nodes represent the compounds and proteins respectively. A. ACE2/DPP4; B. TMPRSS2/SRC; C. FURIN/ADAM17

\section{Supplementary Files}

This is a list of supplementary files associated with this preprint. Click to download.

- Supplementaryfigure1.jpg

- Supplementarytables.zip 